TRANSACTIONS OF THE

AMERICAN MATHEMATICAL SOCIETY

Volume 362, Number 9, September 2010, Pages 4781-4792

S 0002-9947(10)04967-6

Article electronically published on April 26, 2010

\title{
CORESTRICTIONS OF ALGEBRAS AND SPLITTING FIELDS
}

\author{
DANIEL KRASHEN
}

\begin{abstract}
Given a field $F$, an étale extension $L / F$ and an Azumaya algebra $A / L$, one knows that there are extensions $E / F$ such that $A \otimes_{F} E$ is a split algebra over $L \otimes_{F} E$. In this paper we bound the degree of a minimal splitting field of this type from above and show that our bound is sharp in certain situations, even in the case where $L / F$ is a split extension. This gives in particular a number of generalizations of the classical fact that when the tensor product of two quaternion algebras is not a division algebra, the two quaternion algebras must share a common quadratic splitting field.

In another direction, our constructions combined with results of Karpenko (1995) also show that for any odd prime number $p$, the generic algebra of index $p^{n}$ and exponent $p$ cannot be expressed nontrivially as the corestriction of an algebra over any extension field if $n<p^{2}$.
\end{abstract}

\section{INTRODUCTION}

It is a classical fact due to Albert that two quaternion algebras over a field whose tensor product has index at most two must share a common quadratic splitting field. In this paper we give generalizations to this fact in two different directions. On the one hand, we obtain certain generalizations of this statement for algebras of higher degree (see Corollary 4.4 and Example 1.3), which are philosophically similar to, but not intersecting with the results in Kar99. On the other hand, we consider the idea that a pair of algebras may be regarded as an Azumaya algebra over a split étale extension of the form $F \times F$. This leads to analogous results in the case of Azumaya algebras over more general étale extensions (see Theorems 4.2, 4.3 and Examples 1.1 1.2). For example, we derive the following analog of the above fact: given a quadratic field extension $L / F$ and a quaternion division algebra $Q$ over $L$ whose corestriction to $F$ has index at most 2, then $Q$ has a splitting field of the form $E \otimes_{F} L$ for some quadratic field extension $E / L$.

Recall that a finite dimensional algebra $A$ over a field $F$ is called separable if it is an Azumaya algebra over its center, which is a finite dimensional étale extension of $F$. Setting $L=Z(A)$, we may write $L=L_{1} \times \cdots \times L_{m}$ as a product of separable field extensions of $F$, and hence an étale algebra over $F$. Note that such an algebra $A$ may itself be written as a product $A=A_{1} \times \cdots \times A_{m}$, where $A_{i}$ is a central simple $L_{i}$ algebra.

One knows very well the minimal degrees of étale extensions $K / L$ such that $A \otimes_{L} K$ is split: such an extension may be written as $K=K_{1} \times \cdots \times K_{m}$ with $K_{i}$ an extension of $L_{i}$ and one may always find $K$ with $\left[K_{i}: L_{i}\right]=$ ind $A_{i}$. On the other hand, although one knows in principle that there are finite extensions $E / F$ such

Received by the editors May 3, 2007 and, in revised form, November 18, 2008.

2010 Mathematics Subject Classification. Primary 16K20.

(C)2010 American Mathematical Society
Reverts to public domain 28 years from publication 4781 
that $A \otimes_{F} E$ is a split algebra over $L \otimes_{F} E$, (one may easily see this by considering an extension of scalars to an algebraic closure of $F$ ), it may be quite difficult to compute the minimal degree of such an extension. In the case that $L / F$ is a split étale extension, we may write $A=A_{1} \times \cdots \times A_{m}$ and it follows that such an $E$ is exactly a common splitting field for each of the algebras $A_{m}$. Even in this split case, determining a minimal degree for $E / F$ with this property is quite delicate, and an explicit answer is not known in general (however, see Kar99] for various results in this direction).

In this paper we give a construction of étale splitting fields for separable algebras (Theorem 4.2), which we show can provide in some sense optimal bounds on the degrees of splitting extensions (Theorem 4.3, Corollary 4.4, and Propositions 5.2. 5.3). These results generalize the classical fact that two quaternion algebras whose product has index at most two share a common quadratic subfield. Our main results are as follows:

Theorem 4.2. Let $L / F$ be an étale extension of dimension $m$ and $A$ an Azumaya algebra over $L$ of degree $\left(d_{1}, \ldots, d_{m}\right)$ (see Definition [2.6). Let I be the index of $\operatorname{cor}_{L / F} A$ and let $P$ be its period. Let $r$ be the remainder upon dividing $\sum d_{i}-m$ by $I$. Then there exists an étale extension $E / F$ of degree

$$
\frac{\left(d_{1}+\cdots+d_{m}-m\right) !}{\left(d_{1}-1\right) ! \cdots\left(d_{m}-1\right) !} P^{r}
$$

such that $A \otimes_{F} E$ is split as an $L \otimes_{F} E$-algebra.

In the case of prime power degrees, one may say something about the form of this degree:

Theorem 4.3. Let $p$ be a prime number, $L / F$ an étale extension of degree $p^{k}$ and $A$ an Azumaya algebra over $L$ of constant degree $p^{n}$ such that $\operatorname{cor}_{L / F} A$ has index dividing $p^{k}$. Then there exists an étale extension $E / F$ of degree $p^{n\left(p^{k}-1\right)} m$, where $m$ is relatively prime to $p$ such that $A \otimes_{F} E$ is split as an $L \otimes_{F} E$-algebra.

One may always show that the algebra $A$ may be split by such an étale extension $E / F$ with $[E: F]=p^{n p^{k}}$ (see Proposition 2.3). The content of Theorem 4.3 is that after making assumptions about the index, we may in fact do better.

In the case that $L / F$ is a split étale extension, we obtain the following as a Corollary (via Lemma 2.2):

Corollary 4.4. Let $p$ be a prime number, and let $A_{1}, \ldots, A_{p^{k}}$ be central simple algebras of degree $p^{n}$ over a field $F$ such that $A_{1} \otimes \cdots \otimes A_{p^{k}}$ has index dividing $p^{k}$. Then there exists an étale extension $E / F$ of degree $p^{n\left(p^{k}-1\right)} m$, where $m$ is relatively prime to $p$ such that $E$ splits each of the algebras $A_{1}, \ldots, A_{p^{k}}$.

Remark. It follows from the proof of the theorem that the integer $m$ appearing in the statements above may be explicitly expressed as

$$
m=\frac{\left(p^{k}\left(p^{n}-1\right)\right) !}{\left(\left(p^{n}-1\right) !\right)^{p^{k}} p^{n\left(p^{k}-1\right)}} .
$$

The classical case of quaternion algebras corresponds to $p=2, n=1, k=1$ with a split quadratic étale extension. To get a feeling for this result, we provide a few examples for small values. 
Example $1.1(k=n=1, p=2)$. Suppose $L / F$ is a separable quadratic field extension, and suppose $Q$ is a quaternion algebra over $L$ such that $\operatorname{cor}_{L / F} Q$ is not division. Then there exists a quadratic field extension $E / F$ such that $Q \otimes_{F} E$ is split over $L \otimes_{F} E$.

This particular example is already known and may be proved using quadratic form theory, as was pointed out to the author by A. Merkurjev. In particular, one may see this by considering the corestriction of the pure part of the 2-fold Pfister form associated to $Q$ with respect to a linear map $L \rightarrow F$ taking 1 to 0 . Since the result must be isotropic by assumption on the index, the original form must represent an element of the ground field. This implies in the symbol $(a, b)$ defining $Q$ that we may take one of the elements, say $a$, to lie in the ground field, providing us with the splitting field $F(\sqrt{a}) / F$.

Example $1.2(k=n=1$, nonsplit case). Let $p$ be a prime integer. Suppose $L / F$ is a degree $p$ field extension, and suppose $A$ is a degree $p$ central simple $L$-algebra such that $\operatorname{cor}_{L / F} A$ has index dividing $p$. Then there exists an étale extension $E / F$ of degree $p^{p-1} m$ for some $m$ relatively prime to $p$ such that $A \otimes_{F} E$ is split.

Example $1.3\left(k=n=1\right.$, split case). Suppose $A_{1}, \ldots, A_{p}$ are algebras over a field $F$ of prime degree $p$, such that ind $A_{1} \otimes \cdots \otimes A_{p}$ divides $p$. Then there exists an étale extension $E / F$ of degree $p^{p-1} m$ for some $m$ relatively prime to $p$ which splits each algebra $A_{i}$. In particular, for $p=3$ we find that $A$ has a degree $90=9 \cdot 10$ étale splitting extension.

In the split case $n=k=1$ above, this result can be seen to be sharp in the sense of the following propositions:

Proposition 5.2. There exists a field $F$ and central simple $F$-algebras $A_{1}, \ldots, A_{p}$ such that $\operatorname{ind}\left(A_{1} \otimes \cdots \otimes A_{p}\right)=p^{2}$, and every étale extension $E / F$ which splits each algebra $A_{i}$ has $p^{p} \mid[E: F]$.

Proposition 5.3. Let $p$ be a prime number, and choose positive integers $d, n$ with $d<n<p$. Then there exists a field $F$ and central simple $F$-algebras $A_{1}, \ldots, A_{n}$ of degree $p$ such that $\operatorname{ind}\left(A_{1} \otimes \cdots \otimes A_{n}\right)=p^{d}$ and every field extension $E / F$ which splits each algebra $A_{i}$ has $p^{n} \mid[E: F]$.

In Kar95, Karpenko shows that the generic division algebra of index $p^{n}$ and period $p$ is indecomposable for any odd prime $p$. Since an algebra is decomposable if and only if it is a corestriction with respect to a split étale extension (see Lemma 2.2), it makes sense to generalize this result to try to show that the generic division algebra is not a corestriction for any étale extension:

Theorem 3.2. Let $A$ be a generic division algebra of degree $p^{n}$ and period $p$, and let $F$ be the center of $A$. If $n<p^{2}$, then $A$ cannot be written as $\operatorname{cor}_{L / F} B$ for any étale extension $L / F$ and any Azumaya algebra $B$ over $L$.

For $n \geq p^{2}$, for example if $A$ is a division algebra of index $p^{p^{2}}$, the obstruction used in the proof of Theorem 3.2 to show $A$ is not a corestriction vanishes. This raises the question of whether or not such an algebra really may be written as a corestriction. 


\section{Preliminaries}

To begin it will be necessary to develop some machinery for understanding the corestriction of algebras and its relation to the transfer of a scheme.

2.1. Galois twists. Let $F$ be a field, $L / F$ a Galois extension of separable algebras with group $G$ and let $V$ be an $L$-module. For $\sigma$ in $G$, we define a new $L$-module ${ }^{\sigma} V$ as follows. As a set, we define

$$
{ }^{\sigma} V=\left\{{ }^{\sigma}(v) \mid v \in V\right\},
$$

and we endow it with the operations ${ }^{\sigma}(v)+{ }^{\sigma}(w)={ }^{\sigma}(v+w)$ and for $x \in L$, we set $x^{\sigma}(v)={ }^{\sigma}\left(\left(\sigma^{-1} x\right) v\right)$. We let $\phi_{\tau}:{ }^{\sigma} V \rightarrow{ }^{\tau \sigma} V$ denote the natural map $\phi_{\tau}\left({ }^{\sigma}(v)\right)=$ $\tau \sigma(v)$. Note that this map is $\tau$-linear in the sense that $\phi_{\tau}\left(x^{\sigma}(v)\right)=\tau(x) \phi_{\tau}\left({ }^{\sigma}(v)\right)$. One may also check that these maps satisfy $\phi_{\sigma} \phi_{\tau}=\phi_{\sigma \tau}:{ }^{\gamma} V \rightarrow{ }^{\sigma \tau \gamma} V$. By composing with these maps it is easy to check that there is a natural isomorphism of bifunctors $\operatorname{Hom}_{L}\left({ }^{\sigma} V, W\right)=H_{o m}(V, W)$ giving an equivalence between $L$ linear maps from ${ }^{\sigma} V$ to $W$ and $\sigma$-linear maps from $V$ to $W$.

Regarding $V \mapsto{ }^{\sigma} V$ as a functor from the category of $L$-modules to itself, we note that it is additive and monoidal. That is, there are natural isomorphisms ${ }^{\sigma}(V \oplus W)={ }^{\sigma} V \oplus{ }^{\sigma} W$ and ${ }^{\sigma}\left(V \otimes_{L} W\right)={ }^{\sigma} V \otimes_{L}{ }^{\sigma} W$.

This definition may easily be extended to an additive and monoidal functor from the category of $L$-algebras to itself. Suppose $A$ is an $L$-algebra. Then we define the $L$-algebra ${ }^{\sigma} A$ to be the algebra with underlying vector space as defined above, and with the multiplication rule ${ }^{\sigma}(a)^{\sigma}(b)={ }^{\sigma}(a b)$. Note that this amounts to the same thing as taking the same underlying ring and taking the new $L$-structure map $L \rightarrow A$ to be the original one composed with the automorphism $\sigma^{-1}$. As before, the maps $\phi_{\sigma}$ make sense with the same definition and we again have a natural isomorphism $\operatorname{Hom}_{L}\left({ }^{\sigma} A, B\right)=\operatorname{Hom}_{L, \sigma}(A, B)$ of algebra hom sets.

We may similarly extend this definition to schemes defined over $L$ by patching over affine sets. For an $L$-scheme $X$, we denote the resulting scheme by ${ }^{\sigma} X$. By the previous paragraph this amounts to the same thing as taking the same $F$-scheme and composing the structure morphism with the map $\sigma^{-1}: \operatorname{Spec} L \rightarrow \operatorname{Spec} L$. We also obtain a natural isomorphism $\operatorname{Hom}_{L}\left(Y,{ }^{\sigma} X\right)=\operatorname{Hom}_{L, \sigma}(Y, X)$ as before.

2.2. Coset twists. We now extend these constructions to the case where $L / F$ is a separable extension which is not necessarily Galois. Let $E / L$ be a Galois closure of $L / F$ so that $E / F$ is Galois with group $G$ and let $H$ be the subgroup fixing $L$. For an $L$-module $V$ and $\sigma \in G$, we define the coset twist ${ }^{\sigma H} V$ (which will be an $E$-module) as follows. As a set we define

$$
{ }^{\sigma H} V=\frac{\left\{{ }^{\tau}(v) \mid \tau \in \sigma H, v \in V \otimes_{L} E\right\}}{\sim},
$$

where the equivalence relation $\sim$ is defined by letting ${ }^{\tau}(v)={ }^{\gamma}(w)$ if and only if $\gamma^{-1} \tau(v)=w$. Note that $\gamma^{-1} \tau \in H$ and since $v \in V \otimes_{L} E$, elements of $H$ act naturally via the second factor in the tensor product. We define the $E$-module operations by setting ${ }^{\tau}(v)^{\gamma}(w)={ }^{\gamma}\left(\left(\gamma^{-1} \tau(v)\right) w\right)$ and $x^{\tau}(v)={ }^{\tau}\left(\left(\tau^{-1} x\right) v\right)$. Note that the map ${ }^{\sigma}\left(V \otimes_{L} E\right) \rightarrow{ }^{\sigma H} V$ defined by ${ }^{\sigma}(v) \mapsto{ }^{\sigma}(v)$ is an isomorphism.

As before, we have natural morphisms $\phi_{\sigma}:{ }^{\tau H} V \rightarrow{ }^{\sigma \tau H} V$ via $\phi_{\sigma}\left({ }^{\gamma}(v)\right)={ }^{\sigma \gamma}(v)$. Once again, we may check that $\phi_{\sigma} \phi_{\tau}=\phi_{\sigma \tau}$.

This definition may be extended to algebras and varieties, and we make free use of this fact. 
For the ease of exposition for the proof of the following lemma we make the following definition. Given a scheme $X$ and vector bundles $W, V$ over $X$, we call an embedding $W \hookrightarrow V$ admissible if $V / W$ is also a vector bundle (and not merely a coherent sheaf). Equivalently, this says that $W$ is locally a direct summand of $V$. In the case $W \subset V$, we say that $W$ is admissible if the inclusion is admissible.

For an Azumaya algebra $A$ over a ring $L$ of constant degree $n$, we recall (Bla91] or $\mathrm{Kra}$ ) that the $m$ th generalized Severi-Brauer variety $X_{m}(A)$ is the $L$-scheme such that for any $L$-scheme $Y$, we have a natural bijection:

$$
\begin{aligned}
\operatorname{Hom}_{L}\left(Y, X_{m}(A)\right)=\left\{\text { sheaves of rank } m n \text { right ideals } \mathcal{I}<A_{Y} \mid\right. \\
\left.\mathcal{I} \hookrightarrow A_{Y} \text { is admissible }\right\},
\end{aligned}
$$

where we have written $A_{L}$ to denote the fiber product $A \times_{L} Y$ of $A$ regarded as a constant vector bundle over $\operatorname{Spec}(L)$ up to $Y$.

Lemma 2.1. Suppose $A$ is an Azumaya algebra over $L$ and $m<\operatorname{deg} A$. Then there is a natural isomorphism ${ }^{\sigma H} X_{m}(A)=X_{m}\left({ }^{\sigma H} A\right)$.

Proof. We will exhibit this by giving a natural isomorphism between the functors which these schemes represent.

By the description of the scheme $X_{m}(A)$ given above, we have natural isomorphisms

$$
\begin{array}{r}
\operatorname{Hom}_{E}\left(Y,{ }^{\sigma H} X_{m}(A)\right)=\operatorname{Hom}_{E}\left(Y,{ }^{\sigma}\left(X_{m}(A) \times_{L} E\right)\right) \\
=H_{o m}, \sigma\left(Y, X_{m}\left(A \otimes_{L} E\right)\right) \\
=H_{E}\left({ }^{-1} Y, X_{m}\left(A \otimes_{L} E\right)\right) .
\end{array}
$$

This last set may be identified with bundles of ideals $I \subset{ }^{-1} Y \times_{E}\left(A \otimes_{L} E\right)$, and these naturally correspond (via application of the functor ${ }^{\sigma}()$ ) to bundles of ideals $I^{\prime} \subset Y \times_{E}{ }^{\sigma}\left(A \otimes_{L} E\right)$ via $I \mapsto I^{\prime}={ }^{\sigma} I$. We therefore have

$$
\operatorname{Hom}_{E}\left({ }^{\sigma^{-1}} Y, X_{m}\left(A \otimes_{L} E\right)\right)=\operatorname{Hom}_{E}\left(Y, X_{m}\left({ }^{\sigma}(A \otimes E)\right)\right)=\operatorname{Hom}_{E}\left(Y, X_{m}\left({ }^{\sigma H} A\right)\right) \text {, }
$$

as desired.

2.3. Corestriction and transfer. Suppose we have a separable extension of commutative rings $L / F$ and a Galois closure $E / L$ with $G=\operatorname{Gal}(E / F), H$ the subgroup fixing $L$. Given an $L$-algebra $A$, we define

$$
A^{E / L / F}=\underset{\sigma H}{\otimes}{ }^{\sigma H} A
$$

where the tensor product (over the algebra $E$ ) is taken over all cosets of $H$ in $G$. The group $G$ acts naturally on this algebra by defining the action on simple tensors to be

$$
\tau\left(\underset{\sigma H \in G / H}{\otimes} a_{\sigma H}\right)=\underset{\sigma H \in G / H}{\otimes} \phi_{\tau}^{-1}\left(a_{\tau \sigma H}\right) .
$$

This action is semilinear in the sense that $\tau(x b)=\tau(x) \tau(b)$. By the theory of Galois descent, the algebra $A^{E / L / F}$ together with the $G$-action give the descent data for an $F$-algebra, which we call the corestriction (see [Jac96], III.3.13). Explicitly we may define the corestriction of $A$ to be the fixed algebra $\operatorname{cor}_{L / F} A=\left(A^{E / L / F}\right)^{G}$.

Lemma 2.2. Suppose $L=\prod_{i=1}^{k} L_{i}$, and $A$ is an L-algebra. Then writing $A_{i}=$ $L_{i} A$, we have $\operatorname{cor}_{L / F} A=\otimes \operatorname{cor}_{L_{i} / F} A_{i}$. 
Proof. By JJac96, page 151, it follows that for any étale extensions $F \subset K \subset E$, that $\operatorname{cor}_{E / F}=\operatorname{cor}_{K / F} \circ \operatorname{cor}_{E / K}$. Let $K$ be the split étale extension $\prod_{i=1}^{k} F$. It follows easily that $\operatorname{cor}_{E / K}=\prod \operatorname{cor}_{L_{i} / F} A_{i}$. Therefore we only need to verify the statement in the case that $L$ is itself a split algebra.

Now, assuming that $L_{i}=F$ for each $i$, we choose any group $G$ of order $k$, and let it act transitively by permutations of the idempotents of $L$. We may then regard $L / F$ as a $G$-Galois extension, and so $\operatorname{cor}_{L / F} A=\left(A^{L / F}\right)^{G}$. Thinking of $A=\prod A_{i}$, we may write $A^{L / F}$ as a product $\prod_{\sigma \in G}\left(A_{1} \otimes \cdots \otimes A_{k}\right)$, where the elements of $G$ simply permute the terms of the product. An element is fixed by the $G$-action if it is in the image of the diagonal embedding $A_{1} \otimes \cdots \otimes A_{k} \hookrightarrow A^{L / F}$, completing the proof.

Similarly, if $E, L$ and $G$ are as above and we have an $L$-scheme $X$, we define

$$
X^{E / L / F}=\underset{\sigma H}{\times}{ }^{\sigma H} X
$$

with the fiber product taken with respect to $\operatorname{Spec} E$. As before we have a natural Galois action by the group $G$, and so by Galois descent it corresponds to an $F$ scheme, which we denote by $\operatorname{tr}_{L / F} X$.

2.4. A bound on the degree of a splitting field. Let $L$ be a finite commutative separable extension of $F$ and let $A$ be an Azumaya algebra over $L$. We do not assume that the algebra $A$ has constant rank over $L$. We define $X(A)$, the SeveriBrauer variety of $A$, to be the scheme parametrizing right ideals $I$ of $A$ such that for $p \in \operatorname{Spec} L, I_{p}$ is a right ideal of $A_{p}$ of rank $\operatorname{deg} A_{p}$ (see [Gro68]).

Proposition 2.3. Let $A, L, F$ be as above. Then there exists a commutative separable extension $E / F$ with $[E: F]=\prod_{p}\left(\operatorname{deg} A_{p}\right)^{[F(p): F]}$ (where p ranges through the points of $\operatorname{Spec} L$ and $F(p)$ is the residue field of $p$ ) such that $A \otimes_{F} E$ is a split $L \otimes_{F}$ E-algebra.

Proof. Let $K \subset A$ be a maximal commutative separable subalgebra of $A$. Since $A \otimes_{L} K$ is a split algebra over $K$, we obtain a morphism of $L$-schemes $\operatorname{Spec} K \rightarrow$ Spec $X(A)$, and therefore a map

$$
\operatorname{Spec}\left(\operatorname{cor}_{L / F} K\right)=\operatorname{tr}_{L / F}(\operatorname{Spec} K) \rightarrow \operatorname{tr}_{L / F} X(A) .
$$

By the adjointness property of the transfer (see Ser92]), we have

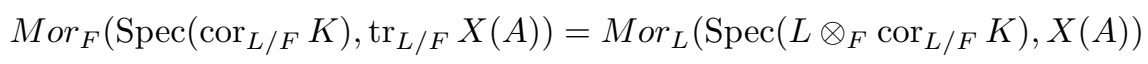

and, in particular, $X(A)$ has an $L \otimes_{F} \operatorname{cor}_{L / F} K$-point. Setting $E=\operatorname{cor}_{L / F} K$, we

now check that $E$ has the stated dimension and note that $A \otimes_{F} E=A \otimes_{L}\left(L \otimes_{F} E\right)$ is split.

\subsection{Twisted Segre embeddings.}

Lemma 2.4. There is a natural closed embedding

$$
\phi_{L / F}^{A}: \operatorname{tr}_{L / F} X(A) \rightarrow X\left(\operatorname{cor}_{L / F} A\right) .
$$

Proof. Let $E / L$ be a Galois closure of $L / F$ with Galois group $G$ acting on $E / F$ and with $H$ the subgroup fixing $L$. We define this morphism by descent by constructing a morphism

$$
\phi: X(A)^{E / L / F} \rightarrow X\left(\operatorname{cor}_{L / F} A\right) \times_{F} E=X\left(\left(\operatorname{cor}_{L / F} A\right) \otimes_{F} E\right)=X\left(A^{E / L / F}\right) .
$$


Note that by Lemma 2.1 we may write

$$
X(A)^{E / L / F}=\prod_{\sigma H}{ }^{\sigma H} X(A)=\prod_{\sigma H} X\left({ }^{\sigma H} A\right) .
$$

We define the map $\phi$ by sending a tuple of ideals indexed by the cosets $G / H$, $\left(I_{\sigma H}\right) \in X(A)^{E / L / F}=\prod_{\sigma H} X\left({ }^{\sigma H} A\right)$ to the tensor product of the ideals $\phi\left(I_{\sigma H}\right)=$ $\otimes I_{\sigma H}$, and note that this commutes with the natural action of the Galois group. Therefore we obtain by descent our desired morphism.

Remark 2.5. In the case that $L / F$ is a split étale extension, we have $A=A_{1} \times$ $\cdots \times A_{m}$ and the map $\phi_{L / F}^{A}$ may be written as the map $X\left(A_{1}\right) \times \cdots \times X\left(A_{m}\right) \rightarrow$ $X\left(A_{1} \otimes \cdots \otimes A_{m}\right)$ given by $\left(I_{1}, \ldots, I_{m}\right) \mapsto I_{1} \otimes \cdots \otimes I_{m}$. This map was investigated by Karpenko in Kar95. In particular, it was shown to be a twisted form of the Segre embedding $\mathbb{P}^{n-1} \times \mathbb{P}^{m-1} \rightarrow \mathbb{P}^{n^{m}-1}$.

Let $X_{L / F}(A)$ denote the image of $\phi_{L / F}^{A}$. We define $\operatorname{deg} X_{L / F}(A)$ to be the degree of the subvariety

$$
X_{L / F}(A)_{\bar{F}} \subset \mathbb{P}^{N},
$$

where $\bar{F}$ is an algebraic closure of $F$. For an algebra $A$ as above, note that $A \otimes_{L} \bar{F}$ is a product of (split) algebras $A_{i}$.

Definition 2.6. Let $A$ be an Azumaya algebra over $L$, where $L / F$ is étale of dimension $m$. Writing $A \otimes_{F} \bar{F}=A_{1} \times \cdots \times A_{m}$ for central simple algebras $A_{i}$, we define $\operatorname{deg} A$ to be the (unordered) list of degrees $\left(\operatorname{deg} A_{1}, \operatorname{deg} A_{2}, \ldots, \operatorname{deg} A_{m}\right)$.

Lemma 2.7. Suppose $L / F$ has degree $m$ and $A / L$ has degree $\left(d_{1}, \ldots, d_{m}\right)$. Then $\operatorname{deg} X_{L / F}(A)$ is the multinomial coefficient

$$
\left(\begin{array}{c}
d_{1}+\cdots+d_{m}-m \\
d_{1}-1, \ldots, d_{m}-1
\end{array}\right)=\frac{\left(d_{1}+\cdots+d_{m}-m\right) !}{\left(d_{1}-1\right) ! \cdots\left(d_{m}-1\right) !} .
$$

Proof. Without loss of generality, we may assume that $F$ is algebraically closed. In this case, we are really considering the embedding of $Y=\mathbb{P}^{d_{1}-1} \times \cdots \times \mathbb{P}^{d_{m}-1}$ into $\mathbb{P}^{d_{1} \cdots d_{m}-1}$ via the Segre embedding, which we will denote by $\phi$. Let $\ell_{i}$ be the pullback of the divisor $\mathcal{O}_{\mathbb{P}^{d_{i}-1}}(1)$ via the natural projection $Y \rightarrow \mathbb{P}^{d_{i}-1}$. Recall that the Chow ring of $Y$ may be written $\mathrm{CH}(Y)=\mathbb{Z}\left[\ell_{1}, \ldots, \ell_{m}\right] /\left(\ell_{i}^{d_{i}}\right)$, and that the Segre embedding is given by the divisor $D=\phi^{*} \mathcal{O}_{\mathbb{P}^{d_{1} d_{2}} \cdots d_{m-1}}(1)=\sum_{i} \ell_{i}$. If we set $d=d_{1}+d_{2}+\cdots+d_{m}-m=\operatorname{dim} Y$, then the degree of the map is therefore given by the degree of $D^{d}$, the top self-intersection of the divisor $D$. By the presentation of the Chow ring of $Y$ given above, it follows that the only term which is nonzero in the multinomial expansion of $D^{d}=\left(\sum_{i} \ell_{i}\right)^{d}$ is the term

$$
\left(\begin{array}{c}
d \\
d_{1}-1, \ldots, d_{m}-1
\end{array}\right) \prod \ell_{i}^{d_{i}-1}
$$

and the fact that $\prod \ell_{i}^{d_{i}-1}$ may be interpreted as the class of a closed point in $Y$ immediately implies the result. 


\section{IS THE GENERIC ALGEBRA A CORESTRICTION?}

Let $A$ be a central simple algebra over $F$ and suppose that $A=\operatorname{cor}_{L / F} B$ for some étale extension $L / F$ of degree $m$ and Azumaya $L$-algebra of constant degree $d$. In particular, this implies that $\operatorname{deg} A=d^{m}$. Since the corestriction map on the level of cohomology cor : $\operatorname{Br}(L) \rightarrow \operatorname{Br}(F)$ is a homomorphism, it follows that $\operatorname{per}(A) \mid \operatorname{per}(B)$ and so $\operatorname{per}(A) \mid d$. It therefore makes sense to ask when the converse holds; namely, if $A$ is an algebra of degree $d^{m}$ and period $d$, when is it a corestriction of an étale extension of degree $m$ ?

A priori, this question is a bit more general than the one of indecomposability, since one knows by Lemma 2.2. that if an algebra is decomposable, it must also be a corestriction (with respect to a split étale extension).

It turns out that in the case $d$ is an odd prime, the arguments of Karpenko from Kar95. generalize nicely to handle corestrictions as well as decomposability. The relevant result which we quote is a special case of a result of Karpenko's:

Lemma 3.1. Let $p$ be a prime number and let $n$ be a positive integer. Let $D\left(p^{n}, p\right)$ be a generic division algebra of degree $p^{n}$ and period $p$ and let $X$ be its Severi-Brauer variety. Then for any cycle $Z \in C H^{k}(X)$, the p-adic valuation of the degree of $Z$ is greater than or equal to the minimum of the following set of numbers:

$$
\left\{i+n-v_{p}(k-i) \mid i=0, \ldots, k-1\right\} \cup\{k\},
$$

where $v_{p}$ denotes the $p$-adic valuation. Furthermore, this remains true even after a prime to $p$ extension.

Proof. See Kar95, Proposition 1.3, and the proof of Theorem 3.1.

Theorem 3.2. Let $A$ be a generic division algebra of degree $p^{n}$ and period $p$, and let $F$ be the center of $A$. If $n<p^{2}$, then $A$ cannot be written as $\operatorname{cor}_{L / F} B$ for any étale extension $L / F$ and any Azumaya algebra $B$ over $L$.

Proof. Suppose we have an algebra $B$ as above, and let $X=X(A)$ be the SeveriBrauer variety of $A$. We claim that there exists some cycle in $X(A)$ which contradicts Lemma 3.1, therefore giving a contradiction. Since this would not be changed by prime to $p$ extensions, we may assume that $F$ has no separable field extensions whose degree is not a power of $p$. Since it follows directly from Kar95, Theorem 3.1 that $A$ is indecomposable, Lemma 2.2 implies that the extension $L$ must in fact be a field and not just an étale algebra. Consequently, the algebra $B$ has constant rank $p^{r}$, and $[L: K]=p^{s}$ for some $r, s$. We therefore also have $p^{n}=\operatorname{deg} A=\left(p^{r}\right)^{p^{s}}=p^{r p^{s}}$. By assumption, $n<p^{2}$ implies that $s=1$.

We may use Lemma 2.4 to embed the variety $\operatorname{tr}_{L / F} X(B)$ as a subvariety in $X$ of codimension $p^{r p}$, and by Lemma 2.7 we find that it has degree

$$
\operatorname{deg} X=\frac{\left(p^{r+1}-p\right) !}{\left(p^{r}-1\right) !^{r}} .
$$

Using Sch84, Lemma 25.5, it follows that $v_{p}(\operatorname{deg} X)=r p-r$. To complete our argument by contradiction, we must now show that the inequality implied by Lemma 3.1 fails. That is, we must show:

$$
\begin{aligned}
v_{p}(\operatorname{deg} X)= & r p-r<i+r p-v_{p}\left(p^{r p}-p^{r}-p-1-i\right), \\
& r p-r<p^{r p}-p^{r}-p-1
\end{aligned}
$$


for $i$ between 0 and $p^{r p}-p^{r}-p-2$. For the second inequality, by grouping terms and rewriting, we see that it is equivalent to

$$
p^{r}\left(p^{r(p-1)-1}\right)>(p-1)(r+1)+2,
$$

and $r(p-1)>1$ (since $p$ is an odd prime) implies $p^{r(p-1)}-1>p-1$, and so it is enough to show that

$$
p^{r} \geq r+1+\frac{2}{p-1}
$$

for which it suffices to show that $p^{r} \geq r+2$. But this is easy to check (note that it holds for $r=1$ and induction on $r$ implies quickly that it holds for all $r$ ).

For the first inequality, we must show:

$$
v_{p}\left(p^{r p}-p^{r}-p-1-i\right)<r+i .
$$

If $i<p^{r}+p+1$, then it follows that $v_{p}\left(p^{r p}-p^{r}-p-1-i\right)<r$, and the inequality follows. Otherwise, $i \geq p^{r}+p+1$ and since $v_{p}\left(p^{r p}-p^{r}-p-1-i\right)$ is always less than $r p$, we must only show $r p<r+p^{r}+p+1$. We start by showing that $p^{r} \geq r p$ for all $r \geq 1$ by induction on $r$. For the induction step, we have:

$$
p^{r+1}=p^{r}+\left(p^{r+1}-p^{r}\right)=p^{r}+p^{r}(p-1) \geq r p+r p(p-1)>r p+p=(r+1) p .
$$

Therefore the inequality may be proved by showing $p+r+1>0$, and we are done.

\section{Splitting FiELDS of SEPARABle ALGEBRAS}

Lemma 4.1. Suppose $A$ is Azumaya over $L$ with $L / F$ étale, and let $E / F$ be another étale extension. Then $A \otimes_{F} E$ is a split algebra over $L \otimes_{F} E$ if and only if the variety $\operatorname{tr}_{L / F} X(A)$ has an E-point.

Proof. By the adjointness property of the transfer (see [Ser92, page 21), we have

$$
H o m_{F}\left(\operatorname{Spec} E, \operatorname{tr}_{L / F} X(A)\right)=H o m_{L}\left(\operatorname{Spec}\left(E \otimes_{F} L\right), X(A)\right) \text {, }
$$

but this in turn implies that

$$
X(A) \times_{\operatorname{Spec} L} \operatorname{Spec}\left(L \otimes_{F} E\right)=X\left(A \otimes_{F} E\right)
$$

has an $L \otimes_{F} E$-point, which implies that $A \otimes_{F} E$ is split.

Theorem 4.2. Let $L / F$ be an étale extension of dimension $m$ and $A$ an Azumaya algebra over $L$ of degree $\left(d_{1}, \ldots, d_{m}\right)$ (see Definition [2.6). Let $I$ be the index of $\operatorname{cor}_{L / F} A$ and let $P$ be its period. Write $\sum d_{i}-m=q I+r$ for positive integers $q, r$ with $r<I$. Then there exists an étale extension $E / F$ of degree

$$
\frac{\left(d_{1}+\cdots+d_{m}-m\right) !}{\left(d_{1}-1\right) ! \cdots\left(d_{m}-1\right) !} P^{r}
$$

such that $A \otimes_{F} E$ is split as an $L \otimes_{F} E$-algebra.

Proof. Let $X=\operatorname{tr}_{L / F} X(A)$ and let $Y=X\left(\operatorname{cor}_{L / F} A\right)$. Since $\operatorname{cor}_{L / F} A$ has index

$I$, there exist cycles $Z^{q I} \subset Y$ such that $Z_{\bar{F}}^{q I}$ is isomorphic to a linear projective subspace of $\mathbb{P}^{d_{1} \cdots d_{m}-1}=Y_{\bar{F}}$ of codimension $q I$ (see Art82]). Also by Art82, there exists a divisor $D \subset Y$ such that $\bar{D}=D_{\bar{F}}$ is in the class $P[H]$, where $H$ is a hyperplane in $\mathbb{P}^{d_{1} \cdots d_{m}-1}$. In particular, using Lemma 2.4 to embed $X$ into $Y$, we find that intersecting a general subspace of the form $Z^{q I}$ with one of the form $D^{r}$ 
will intersect $X$ in a subscheme $C \cong \operatorname{Spec}(E)$, where $E / F$ is an étale extension of degree $(\operatorname{deg} X) P^{r}$.

In particular, this means that $X$ has an $E$-point and our conclusion follows from Lemma 4.1.

Theorem 4.3. Let $p$ be a prime number, $L / F$ an étale extension of degree $p^{k}$ and $A$ an Azumaya algebra over $L$ of constant degree $p^{n}$ such that $\operatorname{cor}_{L / F} A$ has index dividing $p^{k}$. Then there exists an étale extension $E / F$ of degree $p^{n\left(p^{k}-1\right)} m$, where $m$ is relatively prime to $p$ such that $A \otimes_{F} E$ is split as an $L \otimes_{F} E$-algebra.

Proof. Let $X=\operatorname{tr}_{L / F} X(A)$ and let $Y=X\left(\operatorname{cor}_{L / F} A\right)$. As before, we may use Lemma 2.4 to embed $X$ into $Y$ as a closed subscheme. Since $\operatorname{cor}_{L / F} A$ has index $p^{k}$, there exist cycles $Z^{r p^{k}} \subset Y$ such that $Z_{\bar{F}}^{r p^{k}}$ is isomorphic to a linear projective subspace of $\mathbb{P}^{\left(p^{n}\right)^{p^{k}}-1}$ of codimension $r p^{k}$ for any $r$ (see Art82]). In particular, a general subspace of the form $Z^{p^{k+n}-p^{k}}$ will intersect $X$ in a subscheme $C \cong$ $\operatorname{Spec}(E)$, where $E / F$ is an étale extension of degree $\operatorname{deg} X$.

By Lemma 4.1, we therefore need only compute the $p$-adic valuation of $\operatorname{deg} X$ to complete the proof. Using Lemma 2.7, we have:

$$
v_{p}(\operatorname{deg} X)=v_{p}\left(\begin{array}{c}
p^{k+n}-p^{k} \\
p^{n}-1, \ldots, p^{n}-1
\end{array}\right),
$$

and one may show, using, for example, Sch84, Lemma 25.5, that

$$
v_{p}\left(\begin{array}{c}
p^{k+n}-p^{k} \\
p^{n}-1, \ldots, p^{n}-1
\end{array}\right)=\frac{p^{k+n}-p^{k}}{p-1}-n-p^{k}\left(\frac{p^{n}-1}{p-1}-n\right)=n\left(p^{k}-1\right),
$$

as desired.

In the case that $L / F$ is a split étale extension, this gives the following corollary:

Corollary 4.4. Let $p$ be a prime number, and let $A_{1}, \ldots, A_{p^{k}}$ be central simple algebras of degree $p^{n}$ over a field $F$ such that $A_{1} \otimes \cdots \otimes A_{p^{k}}$ has index dividing $p^{k}$. Then there exists an étale extension $E / F$ of degree $p^{n\left(p^{k}-1\right)} m$, where $m$ is relatively prime to $p$ such that $E$ splits each of the algebras $A_{1}, \ldots, A_{p^{k}}$.

Corollary 4.5. Suppose $Q_{1}, Q_{2}$ are quaternion algebras over $F$ and $Q_{1} \otimes Q_{2}$ has index 2 . Then there is a common quadratic splitting extension for $Q_{1}$ and $Q_{2}$.

Proof. This follows immediately from Theorem 4.3 and that in this case $m=1$ by Remark 1].

As a corollary, we present a proof of a result which is known to the experts, but the present context provides a convenient method of proof:

Proposition 4.6. Suppose $Q$ is a quaternion algebra, and $A$ is a division algebra of degree $2 m$ such that $A \otimes Q$ is not division. Then there is a maximal subfield of $A$ which also splits $Q$.

Proof. Let $L=F \times F$ be split quadratic étale, and $B=Q \times A$ as an $L$-algebra. Note that $\operatorname{cor}_{L / F} B=Q \otimes A$ by Lemma 2.2. Let $X=X(B), Y=X(Q \otimes A)$. By section 2, we know that $X$ has dimension $2 m$ and degree $\left(\begin{array}{c}2 m \\ 1,2 m-1\end{array}\right)=2 m$. Since $\operatorname{ind}(Q \otimes A) \mid 2 m$, we have a $Z \subset Y$ in a form of a linear subspace of codimension $2 m$. By intersecting $Z$ with $X$, we obtain a 0 -dimensional subscheme of $2 m$. Using Lemma 4.1. we therefore obtain a splitting field of degree $2 \mathrm{~m}$. 


\section{Counterexamples}

The following lemma will be essential for the construction of counterexamples. In its statement we will use the following notational convention: if $A$ is a central simple $F$-algebra of degree $d$, and $i$ is any integer, we will let $A^{i}$ denote the algebra of degree $d$ which is Brauer equivalent to $A^{\otimes i}$. This algebra is unique up to isomorphism.

Lemma 5.1. Let $d, n$ be positive integers. Then there exists a field $F$ and central simple $F$-algebras $A_{1}, \ldots, A_{n}$ of degree $d$ such that for any $n$-tuple $i_{1}, \ldots, i_{n}$ with $i_{k}$ relatively prime to $d$ for each $k$ the algebra $A_{1}^{i_{1}} \otimes \cdots \otimes A_{n}^{i_{n}}$ is a division algebra.

Proof. See Kar99, proposition III.1.

Proposition 5.2. There exists a field $F$ and central simple $F$-algebras $A_{1}, \ldots, A_{p}$ such that $\operatorname{ind}\left(A_{1} \otimes \cdots \otimes A_{p}\right)=p^{2}$, and every field extension $E / F$ which splits each algebra $A_{i}$ has $p^{p} \mid[E: F]$.

Proof. To begin, choose a field $L$ and central simple algebras $A_{1}, \ldots, A_{p}$ of degree $p$ satisfying the properties described in Lemma [5.1] Let $A=A_{1} \otimes \cdots \otimes A_{p}$ and $A^{\prime}=A_{1} \otimes A_{2} \otimes A_{3}^{2} \otimes A_{4}^{3} \otimes \cdots \otimes A_{p}^{p-1}$. Let $F$ be the function field of the generalized Severi-Brauer variety $X_{p^{2}} A$ of $p^{3}=p \cdot p^{2}$ dimensional right ideals of $A$.

The algebra $A \otimes F$ has index $p^{2}$, but we claim that the algebra $A^{\prime} \otimes F$ has index $p^{p}$. This would prove our claim since if $E / F$ is any common splitting field of $A_{1} \otimes F, \ldots, A_{p} \otimes F$, it would also have to be a splitting field of the algebra $A^{\prime} \otimes F$ and hence have degree divisible by $p^{p}$.

We may compute ind $\left(A^{\prime} \otimes F\right)$ using the index reduction formula of Blanchet ([Bla91] ) as phrased in [MPW96]:

$$
\operatorname{ind}\left(A^{\prime} \otimes F\right)=\underset{1 \leq i \leq p^{p}}{\operatorname{gcd}}\left\{\frac{p^{2}}{\operatorname{gcd}\left\{p^{2}, i\right\}} \text { ind } A^{\prime} \otimes A^{i}\right\}
$$

and by our construction of the algebras $A$ and $A^{\prime}$ we may compute:

$$
\frac{p^{2}}{\operatorname{gcd}\left\{p^{2}, i\right\}} \text { ind } A^{\prime} \otimes A^{i}=\left\{\begin{array}{cc}
p^{2} \cdot p^{p-2} & \text { if } i=p-1, \\
p^{2} \cdot p^{p-1} & \text { if } i \neq p-1 \text { and } p \\
p \cdot p^{p} \text { or } 1 \cdot p^{p} & \text { if } p \mid i
\end{array}\right.
$$

and, in particular, $p^{p}=$ ind $A^{\prime} \otimes F$.

Proposition 5.3. Let $p$ be a prime number, and choose positive integers $d, n$ with $d<n<p$. Then there exists a field $F$ and central simple $F$-algebras $A_{1}, \ldots, A_{n}$ of degree $p$ such that ind $\left(A_{1} \otimes \cdots \otimes A_{n}\right)=p^{d}$ and every field extension $E / F$ which splits each algebra $A_{i}$ has $p^{n} \mid[E: F]$.

Proof. As in the previous lemma, choose a field $L$ and central simple algebras $A_{1}, \ldots, A_{n}$ of degree $p$ satisfying the properties described in Lemma 5.1] Let $A=$ $A_{1} \otimes \cdots \otimes A_{n}$ and $A^{\prime}=A_{1} \otimes A_{2}^{2} \otimes \cdots \otimes A_{p}^{p}$. Let $F$ be the function field of $X_{p^{d}}(A)$.

The algebra $A \otimes F$ has index $p^{d}$, but we claim that the algebra $A^{\prime} \otimes F$ has index $p^{n}$. As in the previous lemma, this would prove our claim. Since the index may only decrease upon scalar extensions, it suffices to show that the algebra $A^{\prime} \otimes L$ has index a multiple of $p^{n}$ for $L$ the function field of $X_{p}(A)$.

We may compute ind $\left(A^{\prime} \otimes F\right)$ :

$$
\operatorname{ind}\left(A^{\prime} \otimes F\right)=\underset{1 \leq i \leq p^{p}}{\operatorname{gcd}}\left\{\frac{p}{\operatorname{gcd}\{p, i\}} \text { ind } A^{\prime} \otimes A^{i}\right\}
$$


and by our construction of the algebras $A$ and $A^{\prime}$ we may compute:

$$
\frac{p}{\operatorname{gcd}\{p, i\}} \text { ind } A^{\prime} \otimes A^{i}=\left\{\begin{array}{cc}
p \cdot p^{n-1} \text { or } p \cdot p^{n} & \text { if } i \neq p, \\
p^{n} & \text { if } i=p
\end{array}\right.
$$

and in particular, $p^{n}=$ ind $A^{\prime} \otimes L$, as claimed.

\section{REFERENCES}

[Art82] M. Artin. Brauer-Severi varieties. In Brauer groups in ring theory and algebraic geometry (Wilrijk, 1981), pages 194-210. Springer, Berlin, 1982. MR657430 (83j:14015)

[Bla91] Altha Blanchet. Function fields of generalized Brauer-Severi varieties. Comm. Algebra, 19(1):97-118, 1991. MR1092553 (92c:14052)

[Gro68] Alexander Grothendieck. Le groupe de Brauer. I. Algèbres d'Azumaya et interprétations diverses. In Dix Exposés sur la Cohomologie des Schémas, pages 46-66. North-Holland, Amsterdam, 1968. MR0244269 (39:5586a)

[Jac96] Nathan Jacobson. Finite-dimensional division algebras over fields. Springer-Verlag, Berlin, 1996. MR1439248(98a:16024)

[Kar95] Nikita A. Karpenko. Torsion in $\mathrm{CH}^{2}$ of Severi-Brauer varieties and indecomposability of generic algebras. Manuscripta Math., 88(1):109-117, 1995. MR1348794 (96g:14007)

[Kar99] Nikita A. Karpenko. Three theorems on common splitting fields of central simple algebras. Israel J. Math., 111:125-141, 1999. MR1710735 (2000k:16020)

[Kra] Daniel Krashen. Zero cycles on homogeneous varieties. submitted for publication, arXiv:math.AG/0501399.

[MPW96] A. S. Merkurjev, I. A. Panin, and A. R. Wadsworth. Index reduction formulas for twisted flag varieties. I. K-Theory, 10(6):517-596, 1996. MR1415325 (98c:16018)

[Sch84] W. H. Schikhof. Ultrametric calculus, volume 4 of Cambridge Studies in Advanced Mathematics. Cambridge University Press, Cambridge, 1984. An introduction to $p$-adic analysis. MR791759 (86j:11104)

[Ser92] Jean-Pierre Serre. Topics in Galois theory. Jones and Bartlett Publishers, Boston, MA, 1992. Lecture notes prepared by Henri Damon [Henri Darmon], With a foreword by Darmon and the author. MR1162313 (94d:12006)

Department of Mathematics, University of Georgia, Athens, Georgia 30602 